\title{
A BERNSTEIN-TYPE INEQUALITY FOR THE JACOBI POLYNOMIAL
}

\author{
YUNSHYONG CHOW, L. GATTESCHI, AND R. WONG
}

(Communicated by Hal L. Smith)

ABsTRACT. Let $P_{n}^{(\alpha, \beta)}(x)$ be the Jacobi polynomial of degree $n$. For $-\frac{1}{2} \leq$ $\alpha, \beta \leq \frac{1}{2}$ and $0 \leq \theta \leq \pi$, it is proved that

$$
\left(\sin \frac{\theta}{2}\right)^{\alpha+\frac{1}{2}}\left(\cos \frac{\theta}{2}\right)^{\beta+\frac{1}{2}}\left|P_{n}^{(\alpha, \beta)}(\cos \theta)\right| \leq \frac{\Gamma(q+1)}{\Gamma\left(\frac{1}{2}\right)}\left(\begin{array}{c}
n+q \\
n
\end{array}\right) N^{-q-\frac{1}{2}},
$$

where $q=\max (\alpha, \beta)$ and $N=n+\frac{1}{2}(\alpha+\beta+1)$. When $\alpha=\beta=0$, this reduces to a sharpened form of the well-known Bernstein inequality for the Legendre polynomial.

\section{INTRODUCTION}

It is well known that the Legendre polynomial $P_{n}(x)$ satisfies the inequality

$$
(\sin \theta)^{\frac{1}{2}}\left|P_{n}(\cos \theta)\right|<\left(\frac{2}{\pi}\right)^{\frac{1}{2}} n^{-\frac{1}{2}}, \quad 0 \leq \theta \leq \pi ;
$$

see $[9,(7.3 .8)$, p. 165]. This inequality is due to S. N. Bernstein, who was the first to determine the least possible constant, $\left(\frac{2}{\pi}\right)^{\frac{1}{2}}$. Recently, by using complex variable methods, Antonov and Holševnikov [1] have shown that the factor $n^{-\frac{1}{2}}$ in $(1.1)$ can be replaced by $\left(n+\frac{1}{2}\right)^{-\frac{1}{2}}$; that is, they have demonstrated the sharper result

$$
(\sin \theta)^{\frac{1}{2}}\left|P_{n}(\cos \theta)\right|<\left(\frac{2}{\pi}\right)^{\frac{1}{2}}\left(n+\frac{1}{2}\right)^{-\frac{1}{2}}, \quad 0 \leq \theta \leq \pi .
$$

Later, Lorch [7] has provided an alternative proof of (1.2), by utilizing essentially a sharpened form of Bernstein's real variable method. Furthermore, in [8] he has shown that the ultraspherical (Gegenbauer) polynomial $P_{n}^{(\lambda)}(x)$ satisfies the inequality

$$
(\sin \theta)^{\lambda}\left|P_{n}^{(\lambda)}(\cos \theta)\right|<2^{1-\lambda}\{\Gamma(\lambda)\}^{-1}(n+\lambda)^{\lambda-1}
$$

Received by the editors September 11, 1992.

1991 Mathematics Subject Classification. Primary 33C.45.

Key words and phrases. Jacobi polynomial, Bernstein inequality, hypergeometric function.

The third author's research was supported by the Natural Sciences and Engineering Research Council of Canada (Grant A7359) and the National Science Council of Taiwan (NSC 81-0208-M001-15). 
for $0<\lambda<1$ and $0 \leq \theta \leq \pi$, which of course improves the customary inequality

$$
(\sin \theta)^{\lambda}\left|P_{n}^{(\lambda)}(\cos \theta)\right|<2^{1-\lambda}\{\Gamma(\lambda)\}^{-1} n^{\lambda-1}, \quad 0 \leq \theta \leq \pi,
$$

given in $[9,(7.33 .5)$, p. 171]. Inequality (1.3) also follows from a more general inequality given by Durand [3, (23)]; see a remark made in [8].

As regards the more general Jacobi polynomial $P_{n}^{(\alpha, \beta)}(x)$, there does not seem to exist an inequality generalizing (1.4). Except for the simple, yet important, estimate

$$
\left|P_{n}^{(\alpha, \beta)}(x)\right| \leq\left(\begin{array}{c}
n+q \\
n
\end{array}\right), \quad-1 \leq x \leq 1, q=\max (\alpha, \beta) \geq-\frac{1}{2},
$$

all we have is the following more recent result of Baratella [2]:

$$
\left(\sin \frac{\theta}{2}\right)^{\alpha+\frac{1}{2}}\left(\cos \frac{\theta}{2}\right)^{\beta+\frac{1}{2}}\left|P_{n}^{(\alpha, \beta)}(\cos \theta)\right| \leq 2.821\left(\begin{array}{c}
n+\alpha \\
n
\end{array}\right) N^{-\alpha-\frac{1}{2}},
$$

where $0 \leq \theta \leq \frac{\pi}{2},-\frac{1}{2} \leq \alpha, \beta \leq \frac{1}{2}$, and

$$
N=n+\frac{\alpha+\beta+1}{2} \text {. }
$$

In view of the reflection formula $[9$, p. 59]

$$
P_{n}^{(\alpha, \beta)}(-x)=(-1)^{n} P_{n}^{(\beta, \alpha)}(x),
$$

Baratella's result in (1.6) can be expressed in the form

$$
\left(\sin \frac{\theta}{2}\right)^{\alpha+\frac{1}{2}}\left(\cos \frac{\theta}{2}\right)^{\beta+\frac{1}{2}}\left|P_{n}^{(\alpha, \beta)}(\cos \theta)\right| \leq 2.821\left(\begin{array}{c}
n+q \\
n
\end{array}\right) N^{-q-\frac{1}{2}}
$$

for $0 \leq \theta \leq \pi$ and $-\frac{1}{2} \leq \alpha, \beta \leq \frac{1}{2}$, where $q=\max (\alpha, \beta)$. In this note, the inequality in (1.6) will be sharpened. Indeed, we shall show that

$$
\left(\sin \frac{\theta}{2}\right)^{\alpha+\frac{1}{2}}\left(\cos \frac{\theta}{2}\right)^{\beta+\frac{1}{2}}\left|P_{n}^{(\alpha, \beta)}(\cos \theta)\right| \leq \frac{\Gamma(q+1)}{\Gamma\left(\frac{1}{2}\right)}\left(\begin{array}{c}
n+q \\
n
\end{array}\right) N^{-q-\frac{1}{2}}
$$

for $0 \leq \theta \leq \pi$ and $-\frac{1}{2} \leq \alpha, \beta \leq \frac{1}{2}$. When $\alpha$ and $\beta$ are restricted to the interval $\left[-\frac{1}{2}, \frac{1}{2}\right]$, it is known that $\Gamma(q+1) \leq \Gamma\left(\frac{1}{2}\right)$. Hence (1.10) improves (1.9) by a factor of 2.821. Baratella's proof is based on an integral equation satisfied by the Jacobi polynomial, whereas our approach is motivated by the complex variable method of Antonov and Holševnikov [1].

If $\alpha=\beta=0$, then our result (1.10) immediately yields (1.2). In the case of ultraspherical polynomials, i.e., when $\alpha=\beta=\lambda-\frac{1}{2}$, we can also show that (1.10) reduces to (1.3), provided that $0<\lambda<\frac{1}{2}$. If $\frac{1}{2}<\lambda<1$, then our result reduces to one which is only slightly weaker than (1.3). For a more detailed discussion of this case, we refer to a remark in Section 4.

\section{A MEHLER-TYPE INTEGRAL}

Let $N$ be given as in (1.7) and put

$$
K(\alpha, \beta, \theta)=\frac{2^{(\alpha+\beta+1) / 2} \Gamma(\alpha+1)}{\Gamma\left(\frac{1}{2}\right) \Gamma\left(\alpha+\frac{1}{2}\right)}(1-\cos \theta)^{-\alpha}(1+\cos \theta)^{-(\alpha+\beta) / 2} .
$$


For $0<\theta<\pi$ and $\operatorname{Re} \alpha>-\frac{1}{2}$, Gasper [4] has given the following Mehler-type integral for the Jacobi polynomial

(2.2)

$$
\begin{aligned}
\frac{P_{n}^{(\alpha, \beta)}(\cos \theta)}{P_{n}^{(\alpha, \beta)}(1)}=K(\alpha, \beta, \theta) \int_{0}^{\theta} & \frac{\cos N \phi}{(\cos \phi-\cos \theta)^{\frac{1}{2}-\alpha}} \\
& \times F\left(\frac{\alpha+\beta}{2}, \frac{\alpha-\beta}{2} ; \alpha+\frac{1}{2} ; \frac{\cos \theta-\cos \phi}{1+\cos \theta}\right) d \phi,
\end{aligned}
$$

where $F(a, b ; c ; z)$ denotes the hypergeometric function and

$$
P_{n}^{(\alpha, \beta)}(1)=\left(\begin{array}{c}
n+\alpha \\
n
\end{array}\right)
$$

Motivated by the method in [1], we consider the remainder

$$
R_{n}(x, \theta)=\sum_{m=n}^{\infty} \frac{P_{m}^{(\alpha, \beta)}(\cos \theta)}{P_{m}^{(\alpha, \beta)}(1)} x^{m} .
$$

In view of the asymptotic behavior of $P_{n}^{(\alpha, \beta)}(\cos \theta)$, the series in (2.4) clearly converges uniformly in $\theta \in(0, \pi)$. Inserting (2.2) in (2.4) gives

$$
\begin{array}{r}
R_{n}(x, \theta)=K(\alpha, \beta, \theta) \int_{0}^{\theta} F\left(\frac{\alpha+\beta}{2}, \frac{\alpha-\beta}{2} ; \alpha+\frac{1}{2} ; \frac{\cos \theta-\cos \phi}{1+\cos \theta}\right) \\
\times \frac{1}{(\cos \phi-\cos \theta)^{\frac{1}{2}-\alpha}} \sum_{m=n}^{\infty}(\cos M \phi) x^{m} d \phi,
\end{array}
$$

where $M=m+\frac{1}{2}(\alpha+\beta+1)$. Since the series under the integral sign can be summed up as

$$
\frac{1}{2} x^{n}\left(\frac{e^{i N \phi}}{1-x e^{i \phi}}+\frac{e^{-i N \phi}}{1-x e^{-i \phi}}\right)
$$

we may rewrite $(2.5)$ in the form

$$
\frac{1}{x^{n}} R_{n}(x, \theta)=\frac{1}{2} K(\alpha, \beta, \theta) \cdot I(\alpha, \beta, \theta),
$$

where

$$
\begin{array}{r}
I(\alpha, \beta, \theta)=\int_{-\theta}^{\theta} F\left(\frac{\alpha+\beta}{2}, \frac{\alpha-\beta}{2} ; \alpha+\frac{1}{2} ; \frac{\cos \theta-\cos \phi}{1+\cos \theta}\right) \\
\times \frac{1}{(\cos \phi-\cos \theta)^{\frac{1}{2}-\alpha}} \cdot \frac{e^{i N \phi}}{1-x e^{i \phi}} d \phi .
\end{array}
$$

To the last integral, we now apply the quadratic transformation [6, p. 251]

$$
\begin{aligned}
F\left(a, b ; a+b+\frac{1}{2} ; z\right)= & \left(\frac{1+\sqrt{1-z}}{2}\right)^{\frac{1}{2}-a-b} \\
& \times F\left(a-b+\frac{1}{2}, b-a+\frac{1}{2} ; a+b+\frac{1}{2} ; \frac{1-\sqrt{1-z}}{2}\right),
\end{aligned}
$$

which is valid for $|\arg (1-z)|<\pi$ and $a+b+\frac{1}{2} \neq 0,-1,-2, \ldots$. In our case, we have

$$
a=\frac{\alpha+\beta}{2}, \quad b=\frac{\alpha-\beta}{2}, \quad \text { and } z=\frac{\cos \theta-\cos \phi}{1+\cos \theta} .
$$


Using the trigonometric identity $1+\cos \phi=2 \cos ^{2}(\phi / 2)$, it is easily seen that $\cos \phi-\cos \theta=2\left[\cos ^{2}(\phi / 2)-\cos ^{2}(\theta / 2)\right]$ and

$$
\frac{1 \pm \sqrt{1-z}}{2}=\frac{\cos (\theta / 2) \pm \cos (\phi / 2)}{2 \cos (\theta / 2)} \text {. }
$$

Consequently, it follows from (2.7) that

$$
\begin{aligned}
I(\alpha, \beta, \theta)= & \frac{1}{2^{1-2 \alpha}[\cos (\theta / 2)]^{\frac{1}{2}-\alpha}} \\
\times \int_{-\theta}^{\theta} F\left(\beta+\frac{1}{2},-\beta+\frac{1}{2}\right. & \left.; \alpha+\frac{1}{2} ; \frac{\cos (\theta / 2)-\cos (\phi / 2)}{2 \cos (\theta / 2)}\right) \\
& \times \frac{1}{[\cos (\phi / 2)-\cos (\theta / 2)]^{\frac{1}{2}-\alpha}} \cdot \frac{e^{i N \phi}}{1-x e^{i \phi}} d \phi .
\end{aligned}
$$

(Note that $\operatorname{Re} \alpha>-\frac{1}{2}$ and hence $\alpha+\frac{1}{2} \neq 0,-1,-2, \ldots$.) Since $1-\cos \theta=$ $2 \sin ^{2}(\theta / 2)$, equation (2.1) can be written as

$$
K(\alpha, \beta, \theta)=\frac{\Gamma(\alpha+1)}{\Gamma\left(\frac{1}{2}\right) \Gamma\left(\alpha+\frac{1}{2}\right)} 2^{\frac{1}{2}-\alpha}\left(\sin \frac{\theta}{2}\right)^{-2 \alpha}\left(\cos \frac{\theta}{2}\right)^{-\alpha-\beta} .
$$

A combination of (2.6), (2.11) and (2.12) gives

$$
\frac{1}{x^{n}} R_{n}(x, \theta)=2^{\alpha-\frac{3}{2}} \frac{\Gamma(\alpha+1)}{\Gamma\left(\frac{1}{2}\right) \Gamma\left(\alpha+\frac{1}{2}\right)}\left(\sin \frac{\theta}{2}\right)^{-2 \alpha}\left(\cos \frac{\theta}{2}\right)^{-\beta-\frac{1}{2}} I^{*}(\alpha, \beta, \theta),
$$

where

$$
\begin{aligned}
I^{*}(\alpha, \beta, \theta)=\int_{-\theta}^{\theta} & F\left(\beta+\frac{1}{2},-\beta+\frac{1}{2} ; \alpha+\frac{1}{2} ; \frac{\cos (\theta / 2)-\cos (\phi / 2)}{2 \cos (\theta / 2)}\right) \\
& \times \frac{1}{[\cos (\phi / 2)-\cos (\theta / 2)]^{\frac{1}{2}-\alpha}} \cdot \frac{e^{i N \phi}}{1-x e^{i \phi}} d \phi .
\end{aligned}
$$

So far the only conditions which we require are

$$
0<\theta<\pi \text { and } \operatorname{Re} \alpha>-\frac{1}{2} .
$$

Now we deform the path of integration in (2.14) into two vertical lines $\operatorname{Re} \phi=\theta$ and $\operatorname{Re} \phi=-\theta$. This can be achieved by showing that the contribution from the horizontal line segment, $\operatorname{Im} \phi=T$ and $-\theta \leq \operatorname{Re} \phi \leq \theta$, approaches zero as $T \rightarrow+\infty$. Thus we have

$$
I^{*}(\alpha, \beta, \theta)=i I_{-}(\alpha, \beta, \theta)-i I_{+}(\alpha, \beta, \theta),
$$

where

$$
\begin{aligned}
I_{ \pm}(\alpha, \beta, \theta)=\int_{0}^{\infty} F(\beta+ & \left.\frac{1}{2},-\beta+\frac{1}{2} ; \alpha+\frac{1}{2} ; \frac{\cos \frac{1}{2} \theta-\cos \frac{1}{2}( \pm \theta+i \tau)}{2 \cos \frac{1}{2} \theta}\right) \\
& \times \frac{e^{i N( \pm \theta+i \tau)}}{\left[\cos \frac{1}{2}( \pm \theta+i \tau)-\cos \frac{1}{2} \theta\right]^{\frac{1}{2}-\alpha}} \frac{d \tau}{1-x e^{i( \pm \theta+i \tau)}} .
\end{aligned}
$$


The validity of $(2.17)$ requires that

$$
\alpha+\beta>0 \text { and } \beta>-\frac{1}{2}
$$

see the conditions for equation (3.1) below. Since the hypergeometric function $F(a, b ; c ; z)$ is symmetric in $a$ and $b,(2.17)$ is also valid under the conditions

$$
\alpha>\beta>-\frac{1}{2} \text {. }
$$

One could have proceeded with the deformation of contour directly from the integral in (2.7), but this would yield a smaller region of validity for the parameters $\alpha$ and $\beta$.

Our next step is to estimate the integrals in (2.17).

\section{Proof of (1.10)}

We first recall the integral representation $[6$, p. 239]

$$
F(a, b ; c ; z)=\frac{\Gamma(c)}{\Gamma(b) \Gamma(c-b)} \int_{0}^{1} t^{b-1}(1-t)^{c-b-1}(1-z t)^{-a} d t,
$$

where $\operatorname{Re} c>\operatorname{Re} b>0$ and $|\arg (1-z)|<\pi$. If $\operatorname{Re} z<0$ and $\operatorname{Re} a>0$, then it is easily seen from $(3.1)$ that $F(a, b ; c ; z)$ is bounded by 1 in absolute value. Since the real part of

$$
\frac{\cos \frac{1}{2} \theta-\cos \frac{1}{2}( \pm \theta+i \tau)}{2 \cos \frac{1}{2} \theta}
$$

is negative for $\tau>0$, it follows that

$$
\left|F\left(\beta+\frac{1}{2},-\beta+\frac{1}{2} ; \alpha+\frac{1}{2} ; \frac{\cos \frac{1}{2} \theta-\cos \frac{1}{2}( \pm \theta+i \tau)}{2 \cos \frac{1}{2} \theta}\right)\right| \leq 1
$$

either under the conditions

$$
\alpha+\beta>0 \text { and } \frac{1}{2}>\beta>-\frac{1}{2},
$$

or under the conditions

$$
\alpha>\beta \quad \text { and } \quad \frac{1}{2}>\beta>-\frac{1}{2} .
$$

Applying (3.2) to (2.17), we obtain

$$
\left|I_{ \pm}(\alpha, \beta, \theta)\right| \leq \int_{0}^{\infty} \frac{e^{-N \tau}}{\left|\cos \frac{1}{2}( \pm \theta+i \tau)-\cos \frac{1}{2} \theta\right|^{\frac{1}{2}-\alpha}} \cdot \frac{d \tau}{\mid 1-x e^{i( \pm \theta+i \tau) \mid}} .
$$

Simple calculation shows

$$
\left|\cos \left(\frac{ \pm \theta+i \tau}{2}\right)-\cos \frac{\theta}{2}\right|^{2}=4 \sinh ^{2} \frac{\tau}{4} \cdot\left(\sinh ^{2} \frac{\tau}{4}+\sin ^{2} \frac{\theta}{2}\right) .
$$

Hence

$$
\left|\cos \left(\frac{ \pm \theta+i \tau}{2}\right)-\cos \frac{\theta}{2}\right| \geq 2 \sinh \frac{\tau}{4} \sin \frac{\theta}{2} \geq \frac{\tau}{2} \sin \frac{\theta}{2}, \quad 0<\theta<\pi .
$$

Since

$$
\lim _{x \rightarrow 0}\left|1-x e^{i( \pm \theta+i \tau)}\right|=1, \quad 0<\theta<\pi,
$$


coupling (3.4) and (3.5) yields

$$
\lim _{x \rightarrow 0}\left|I_{ \pm}(\alpha, \beta, \theta)\right| \leq\left(\frac{1}{2} \sin \frac{\theta}{2}\right)^{\alpha-\frac{1}{2}} \frac{\Gamma\left(\alpha+\frac{1}{2}\right)}{N^{\alpha+\frac{1}{2}}},
$$

provided that either

$$
\alpha+\beta>0, \quad \beta>-\frac{1}{2}, \quad \text { and } \quad-\frac{1}{2}<\alpha<\frac{1}{2},
$$

or

$$
\alpha>\beta, \quad \beta>-\frac{1}{2}, \quad \text { and } \quad-\frac{1}{2}<\alpha<\frac{1}{2} \text {. }
$$

A combination of (2.13), (2.16), and (3.6) gives

$$
\lim _{x \rightarrow 0} \frac{1}{x^{n}}\left|R_{n}(x, \theta)\right| \leq\left(\sin \frac{\theta}{2}\right)^{-\alpha-\frac{1}{2}}\left(\cos \frac{\theta}{2}\right)^{-\beta-\frac{1}{2}} \frac{\Gamma(\alpha+1)}{\Gamma\left(\frac{1}{2}\right) N^{\alpha+\frac{1}{2}}} .
$$

From (2.4), (2.3), and (3.8) it follows that

$$
\left(\sin \frac{\theta}{2}\right)^{\alpha+\frac{1}{2}}\left(\cos \frac{\theta}{2}\right)^{\beta+\frac{1}{2}}\left|P_{n}^{(\alpha, \beta)}(\cos \theta)\right| \leq \frac{\Gamma(\alpha+1)}{\Gamma\left(\frac{1}{2}\right)}\left(\begin{array}{c}
n+\alpha \\
n
\end{array}\right) N^{-\alpha-\frac{1}{2}} .
$$

Let $\theta=\pi-\phi$. By (3.9) and the reflection formula (1.8),

$$
\left(\cos \frac{\phi}{2}\right)^{\alpha+\frac{1}{2}}\left(\sin \frac{\phi}{2}\right)^{\beta+\frac{1}{2}}\left|P_{n}^{(\beta, \alpha)}(\cos \phi)\right| \leq \frac{\Gamma(\alpha+1)}{\Gamma\left(\frac{1}{2}\right)}\left(\begin{array}{c}
n+\alpha \\
n
\end{array}\right) N^{-\alpha-\frac{1}{2}} .
$$

Replacing $\phi$ by $\theta$ and reversing the roles of $\alpha$ and $\beta$, we have

$$
\left(\sin \frac{\theta}{2}\right)^{\alpha+\frac{1}{2}}\left(\cos \frac{\theta}{2}\right)^{\beta+\frac{1}{2}}\left|P_{n}^{(\alpha, \beta)}(\cos \theta)\right| \leq \frac{\Gamma(\beta+1)}{\Gamma\left(\frac{1}{2}\right)}\left(\begin{array}{c}
n+\beta \\
n
\end{array}\right) N^{-\beta-\frac{1}{2}},
$$

either under the conditions

$$
\beta+\alpha>0, \quad \alpha>-\frac{1}{2}, \quad \text { and }-\frac{1}{2}<\beta<\frac{1}{2},
$$

or under the conditions

$$
\beta>\alpha, \quad \alpha>-\frac{1}{2}, \quad \text { and } \quad-\frac{1}{2}<\beta<\frac{1}{2} .
$$

The desired inequality now follows from (3.9) and (3.10), using the set of conditions given in (3.7b) and (3.11b). The special case $\alpha=\beta$ can be treated by a limiting argument.

\section{REMARKS}

1. If $\alpha$ and $\beta$ are both restricted to the interval $\left(-\frac{1}{2}, \frac{1}{2}\right)$, then the two sets of conditions in (3.7a) and (3.11a) are the same. Hence inequality (1.10) holds with $q=\min (\alpha, \beta)$, instead of $q=\max (\alpha, \beta)$. However, the validity of this stronger result is only in half of the unit square $\left(-\frac{1}{2}, \frac{1}{2}\right) \times\left(-\frac{1}{2}, \frac{1}{2}\right)$, namely, $\alpha+\beta>0$. It would be desirable to extend (1.10) to allow $\alpha+\beta<0$, $\alpha, \beta \in\left(-\frac{1}{2}, \frac{1}{2}\right)$.

2. If $\alpha=\beta$, then the Jacobi polynomial $P_{n}^{(\alpha, \beta)}(x)$ reduces to the ultraspherical polynomial $P_{n}^{(\lambda)}(x), \lambda=\alpha+\frac{1}{2}$. More precisely, we have

$$
P_{n}^{(\lambda)}(x)=\frac{\Gamma(\alpha+1)}{\Gamma(2 \alpha+1)} \cdot \frac{\Gamma(n+2 \alpha+1)}{\Gamma(n+\alpha+1)} P_{n}^{(\alpha, \alpha)}(x), \quad \alpha=\lambda-\frac{1}{2}
$$


see $[9,(4.7 .1)$, p. 80$]$. Since $P_{n}^{(\alpha, \beta)}(x)$ is continuous in $\beta$, we may let $\beta$ approach $\alpha$ in (3.9). In view of (4.1) and the duplication formula for the gamma function, this gives

$$
(\sin \theta)^{\lambda}\left|P_{n}^{(\lambda)}(\cos \theta)\right| \leq \frac{2^{1-\lambda}}{\Gamma(\lambda)} \cdot \frac{\Gamma(n+2 \lambda)}{\Gamma(n+1)}(n+\lambda)^{-\lambda} .
$$

Lorch's result (1.3) now follows from the inequality

$$
\frac{\Gamma(n+2 \lambda)}{\Gamma(n+1)}<\frac{1}{(n+\lambda)^{1-2 \lambda}}
$$

provided that $0<2 \lambda<1$; see [8, (8)] or [5, (1.3)]. If $1<2 \lambda<2$, i.e., $0<\alpha<\frac{1}{2}$, then by the inequality $[8,(10)]$

$$
\frac{\Gamma(n+2 \lambda)}{\Gamma(n+1)}<\frac{1}{(n+2 \lambda)^{1-2 \lambda}}
$$

we have from (4.2)

$$
(\sin \theta)^{\lambda}\left|P_{n}^{(\lambda)}(\cos \theta)\right| \leq \frac{2^{1-\lambda}}{\Gamma(\lambda)} \cdot \frac{(n+2 \lambda)^{2 \lambda-1}}{(n+\lambda)^{\lambda}},
$$

which is only slightly weaker than (1.3).

\section{REFERENCES}

1. V. A. Antonov and K. V. Holševnikov, An estimate of the remainder in the expansion of the generating function for the Legendre polynomials (Generalization and improvement of Bernstein's inequality), Vestnik Leningrad Univ. Math. 13 (1981), 163-166.

2. P. Baratella, Bounds for the error term in Hilb formula for Jacobi polynomials, Atti Accad. Sci. Torino Cl. Sci. Fis. Mat. Natur. 120 (1986), 207-233.

3. L. Durand, Nicholson-type integrals for products of Gegenbauer functions and related topics, Theory and Applications of Special Functions (R. Askey, ed.), Academic Press, New York, 1975, pp. 353-374.

4. G. Gasper, Formulas of the Dirichlet-Mehler type, Fractional Calculus and Its Applications (B. Ross, ed.), Lecture Notes in Math., vol. 457, Springer-Verlag, New York, pp. 207-215.

5. D. Kershaw, Some extensions of Gautschi's inequalities for the gamma function, Math. Comp. 41 (1983), 607-611.

6. N. N. Lebedev, Special functions and their applications, Prentice-Hall, Englewood Cliffs, NJ, 1965.

7. L. Lorch, Alternative proof of a sharpened form of Bernstein's inequality for Legendre polynomials, Appl. Anal. 14 (1983), 237-240.

8. __ Inequalities for ultraspherical polynomials and the gamma function, J. Approx. Theory 40 (1984), 115-120.

9. G. Szegö, Orthogonal polynomials, 4th ed., Colloq. Publ., vol. 23, Amer. Math. Soc., Providence, RI, 1975.

Institute of Mathematics, Academia Sinica, Taipei, Taiwan

Department of Mathematics, University of Torino, Torino, Italy

Department of Applied Mathematics, University of Manitoba, Winnipeg, Manitoba, CANADA R3T 2N2

Current address: Department of Mathematics, City Polytechnic of Hong Kong, 83 Tat Chee Avenue, Kowloon, Hong Kong

E-mail address: mawong@cphkvx.cphk.hk 